\title{
One or more elastographic methods for liver fibrosis assessment?
}

\author{
Ioan Sporea, MD, PhD
}

Department of Gastroenterology and Hepatology, "Victor Babes" University of Medicine and Pharmacy Timisoara, Romania

In every day practice many patients with chronic hepatopathies of various aetiologies need to be evaluated. How do we do this? Traditionally, liver biopsy is the classical assessment method in chronic liver diseases. But in the last decade, development of non-invasive evaluation methods in liver diseases and patients' access to the newest medical information by means of internet, have made them to ask questions about how this evaluation can be done.

Transient Elastography (TE) (FibroScan) is a validated method in many European countries (starting with France), and the European Guidelines (EASL) of HCV treatment recommend this method for non-invasive evaluation of liver fibrosis [1]. Why? Because there are a great many papers showing a good correlation between the severity of liver fibrosis and TE measurements, especially for advanced fibrosis in HCV patients. Furthermore, three meta-analyses have proved a good correlation between histology and this method [2-4]. This is why TE is extensively used in many European countries and in Asia, and more recentlyit was approved by FDA in the United States. Following the good results of TE in HCV chronic infection, this method was used for HBV infection, NASH or others chronic hepatopathies. In Romania we have more than 25 FibroScan units extensively used in daily practice. This is why the number of liver biopsies have decreased dramatically in Romania in the last years [5]. The criticism for TE is that valid measurements can be obtained in only $70-85 \%$ of the patients using only the $M$ probe [6,7], but this number can be increased to

Received Accepted

Med Ultrason

2015, Vol. 17, No 2, 137-138

Corresponding author: Prof. Ioan Sporea, $\mathrm{MD}, \mathrm{PhD}$

13, Snagov Street, 300482 Timisoara, Romania

Phone: +40 256309455

Fax: + 40256488003

E-mail: isporea@umft.ro more than $90 \%$ using the XL probe. Other criticisms are the variability of measurements during time [8], the high maintenance cost of the system, with bi-annual calibration of the probe (increasing the initial cost of the FibroScan machine), and the blind modality for liver evaluation.

During the last years, other ultrasound based elastographic methods, with a real time visualization of the liver parenchyma, were tested. Shear wave elastographic (SWE) methods (point SWE and 2D SWE) [9] are now implemented in many ultrasound machines (Siemens, Philips, Aixplorer, GE, Toshiba). Which are the advantages of such systems? Being implemented in standard ultrasound machines, these devices can also be used for daily ultrasound examination, to perform Contrast Enhanced ultrasound (CEUS) or Doppler evaluation. Thus we have a "one shop stop" in our hand. We can start with a standard ultrasound examination to find signs of chronic hepatopathy (liver or spleen enlargement, steatosis, heterogeneity of liver structure, signs of portal hypertension, etc), to find or exclude tumoral masses in the liver, or ascites and finally to immediately perform an elastographic evaluation, choosing the measurement place by gray scale ultrasound. Thus, Shear Wave Elastographic methods (point SWE or 2D SWE) seem to be ideal for daily practice. Another advantage is their price, roughly the same as using FibroScan, but they do not need biannual recalibration and also there is no need for a long training period for the user.

The next question is if, at this moment, there are enough scientific arguments to validate these methods for daily practice (evidence based medicine). Large cohorts of patients evaluated by these systems and meta-analyses are needed for validation. A study performed in a cohort of more than 1000 patients showed a good correlation between point SWE (Acoustic Radiation Force Impulse - ARFI Elastography) and histology, both in HBV and HCV chronic hepatitis. It also found that ARFI and TE 
One or more elastographic methods for liver fibrosis assessment?

seem to be equal for liver fibrosis evaluation [10]. Furthermore, two meta-analyses found good AUROCs values for liver fibrosis assessment and non-inferiority in comparison with TE $[11,12]$.

2D SWE, a real time elastographic method whose results are displayed both as colour coded images and as numeric values, despite the fact that is relatively new on the market, showed good performance in estimating liver stiffness. Starting with the first papers that found a good correlation between this method and histology [13] and also with TE [14] and finishing with the last study recently presented at the EASL meeting in Vienna [15], all published data showed the non-inferiority or even the superiority of 2D SWE as compared with a validated method for non-invasive assessment of fibrosis, TE. In the last paper on 2D SWE more than 1000 patients were included, in which 2D SWE was compared with liver biopsy and TE. The conclusion of this study was that 2D SWE showed a good to excellent performance for non-invasive assessment of liver fibrosis [15]. Also, data from an unpublished meta-analysis regarding 2D SWE showed a very good correlation with fibrosis.

Recent papers, such as the Romanian National Guideline and clinical recommendationon liver elastography [16] and WFUMB Guidelines [17], confirmed the practical utility of all three SWE methods for liver fibrosis assessment.

Finally, I believe that it is the time to validate for clinical practice, besides TE, other SWE methods, such as point SWE and 2D SWE. This will enable physicians to evaluate a larger cohort of patients easily, inexpensively and non traumatically, immediately after a standard ultrasound evaluation.

Let's do it! Let's validate all three types of SWE as soon as possible, because the scientific body of evidence is very strong now!

\section{References}

1. European Association for the Study of the Liver. EASL Clinical Practice Guidelines: management of hepatitis C virus infection. J Hepatol 2011; 55: 245-264.

2. Talwalkar JA, Kurtz DM, Schoenleber SJ, West CP, Montori VM. Ultrasound-based transient elastography for the detection of hepatic fibrosis: systematic review and metaanalysis. Clin Gastroenterol Hepatol 2007; 5: 1214-1220.

3. Friedrich-Rust M, Ong MF, Martens S, et al. Performance of transient elastography for the staging of liver fibrosis: a meta-analysis. Gastroenterology 2008; 134: 960-974.

4. Tsochatzis EA, Gurusamy KS, Ntaoula S, Cholongitas E, Davidson BR, Burroughs AK. Elastography for the diagno- sis of severity of fibrosis in chronic liver disease: a metaanalysis of diagnostic accuracy. J Hepatol 2011; 54: 650659.

5. Sporea I, Popescu A, Gheorghe L, CijevschiPrelipcean C, Spârchez Z, Voiosu R. "Quo vadis" liver biopsy? A multicentre Romanian study regarding the number of liver biopsies performed for chronic viral hepatitis. J Gastrointestin Liver Dis 2012; 21: 326.

6. Castera L, Foucher J, Bernard PH, et al. Pitfalls of liver stiffness measurement: a 5-year prospective study of 13,369 examinations. Hepatology 2010; 51: 828-835.

7. Șirli R, Sporea I, Bota S, Jurchiş A. Factors influencing reliability of liver stiffness measurements using transient elastography (M-probe)-monocentric experience. Eur J Radiol 2013; 82: e313-e316.

8. Nascimbeni F, Lebray P, Fedchuk L, et al; LIDO Study Group. Significant Variations in Elastometry Measurements Made Within Short-term in Patients With Chronic Liver Diseases. Clin Gastroenterol Hepatol 2015; 13: 763-771.e6.

9. Cosgrove D, Piscaglia F, Bamber J, et al. EFSUMB guidelines and recommendations on clinical use of ultrasound elastography. Part 2: Clinical applications. Ultraschall Med 2013; 34: 238-253.

10. Sporea I, Bota S, Peck-Radosavljevic M, et al. Acoustic Radiation Force Impulse elastography for fibrosis evaluation in patients with chronic hepatitis C: an international multicenter study. Eur J Radiol 2012; 81: 4112-4118.

11. Bota S, Herkner H, Sporea I, et al. Meta-analysis: ARFI elastography versus transient elastography for the evaluation of liver fibrosis. Liver Int 2013; 33: 1138-1147.

12. Nierhoff J, Chávez Ortiz AA, Herrmann E, Zeuzem S, Friedrich-Rust M. The efficiency of acoustic radiation force impulse imaging for the staging of liver fibrosis: a metaanalysis. Eur Radiol 2013; 23: 3040-3053.

13. Bavu E, Gennisson JL, Couade M, et al. Noninvasive in vivo liver fibrosis evaluation using supersonic shear imaging: a clinical study on 113 hepatitis $\mathrm{C}$ virus patients. Ultrasound Med Biol 2011; 37: 1361-1373.

14. Ferraioli G, Tinelli C, Dal Bello B, Zicchetti M, Filice G, Filice C; Liver Fibrosis Study Group. Accuracy of real-time shear wave elastography for assessing liver fibrosis in chronic hepatitis C: A pilot study. Hepatology 2012; 56: 2125-2133.

15. Herrmann E, de Lédinghen V, Cassinotto C, et al. 2D-Shear Wave Elastography is equivalent or superior to Transient Elastography for liver fibrosis assessment: results from an individual patient data based meta-analysis. Accepted Abstract RS-3186, EASL 2015.

16. Sporea I, Bota S, Săftoiu A, et al. Romanian national guidelines and practical recommendations on liver elastography. Med Ultrason 2014; 16: 123-138.

17. Ferraioli G, Filice C, Castera L, et al. WFUMB Guidelines and Recommendations for Clinical Use of Ultrasound Elastography Part 3: Liver. Ultrasound Med Biol 2015 Mar 19. doi: 10.1016/j.ultrasmedbio.2015.03.007. 